\title{
The association of MPO gene promoter polymorphisms with Alzheimer's disease risk in Chinese Han population
}

\author{
Wenzhen $\mathrm{Ji}^{1, *}$ and Yu Zhang ${ }^{2, *}$ \\ ${ }^{1}$ Department of Neurology, Tianjin Huanhu Hospital, Tianjin Key Laboratory of Cerebrovascular and Neurodegenerative \\ Diseases, Tianjin 300000, China \\ ${ }^{2}$ Division of Medical Affairs, Tianjin Huanhu Hospital, Tianjin Key Laboratory of Cerebrovascular and Neurodegenerative \\ Diseases, Tianjin 300000, China \\ *Co-first author: Wenzhen Ji and Yu Zhang \\ Correspondence to: Wenzhen Ji, email: oiwdfg@yeah.net \\ Yu Zhang, email: io23rdgd@126.com \\ Keywords: Alzheimer's disease; MPO; polymorphisms; plasma concentration \\ Received: June 22, $2017 \quad$ Accepted: September 20, $2017 \quad$ Published: November 06, 2017 \\ Copyright: $\mathrm{Ji}$ et al. This is an open-access article distributed under the terms of the Creative Commons Attribution License 3.0 \\ (CC BY 3.0), which permits unrestricted use, distribution, and reproduction in any medium, provided the original author and source \\ are credited.
}

\section{ABSTRACT}

Aim: The objective of this study was to explore the genetic association of myeloperoxidase (MPO) gene polymorphisms with risk of Alzheimer19;s disease (AD).

Methods: Blood samples were collected from 116 AD patients and 134 age and gender matched healthy individuals. Polymerase chain reaction-restriction fragment length polymorphism (PCR-RFLP) was utilized to confirm MPO polymorphisms in promoter region. Plasma concentration of MPO was detected by enzyme-linked immuno sorbent assay. Genotype distributions of MPO polymorphisms were compared by $\mathrm{X}^{2}$ test between the two groups. The status of linkage disequilibrium between MPO two polymorphisms was detected using Haploview. MPO concentrations were analyzed by non-parametric test.

Results: MPO rs2333227 polymorphism was positively associated with AD risk, especially under the AA+GA vs. GG and A vs. G genetic models $(P=0.042$, $O R=1.719,95 \% C I=1.017-2.906 ; P=0.041, O R=1.582,95 \% C I=1.016-2.463)$. While, rs34097845 polymorphism significantly decreased the risk of $A D$, particularly $G A$ and AA+GA genotypes $(P=0.048, O R=0.555,95 \% C I=0.308-0.998 ; P=0.042, O R=0.552$, $95 \% \mathrm{CI}=0.310-0.983)$. In addition, rs2333227 genotypes affected the plasma concentration of MPO. But for rs34097845 polymorphism, only GA genotype exhibited significant association with MPO concentration.

Conclusion: Polymorphisms in the promoter region of MPO distinctly contribute to AD risk possibly through regulating MPO concentration. Present results should be confirmed by further studies.

\section{INTRODUCTION}

Alzheimer19; s disease (AD) is one of the most common neurodegenerative diseases in old people. It is a long lasting disorder with irreversible process [1]. Its incidence is increasing with age, and the symptoms are becoming serious over the time [2]. The clinical symptoms of $\mathrm{AD}$ are characterized by short-term memory loss, language disorder, and behavioural issues [3]. The therapy and nursing of $\mathrm{AD}$ cause heavy economic burden and life stress to patients' family and the society $[4,5]$. However, the etiology of AD is not well understood. A lot of studies indicate that the major pathological characteristics of $\mathrm{AD}$ include abnormal deposition of amyloid beta $(\mathrm{A} \beta)$ and tau protein in brain, the loss of synapses and neurons, and the neurofibrillary tangles [6-9]. Besides, other researches find 
some defects may be also involved in the development of $\mathrm{AD}$, such as vasculopathy, lipidosis, autophagy and lysosomal defects, etc [10-13]. AD is a complex disease which is regulated by the genetic and environmental factors [14]. It is generally considered that the genetic factors, especially the key genes associated with the pathological characteristics, play a crucial role in AD [15].

Myeloperoxidase (MPO), a heme protein, is abundant in neutrophil granulocytes and monocytes [16]. MPO has the activity of peroxidase and lysosomal enzyme [17]. MPO mediates the lipid metabolism [18], inflammatory responses [19], tissue damages [20], lysosomal pathway [21], and oxidative damage [22]. Abnormal expression of MPO may result in various disorders. It has been indicated that MPO was potentially associated with several neurodegenerative diseases [23]. For example, Tzikas et al. found that the plasma level of MPO was significantly higher in AD patients than that in healthy controls [24]. In human, MPO protein is encoded by $M P O$ gene which is located in $17 \mathrm{q} 23.1$. Polymorphisms in $M P O$ gene could alter the structure or expression level of the corresponding protein [25]. For instance, rs2333227 $(-463 \mathrm{~A} / \mathrm{G})$ polymorphism is located at the promoter region of $M P O$ gene, which can bind with SP1 site. Alterations in the locus (463A) may influence the transcription activity of the gene, thus impacting the level of MPO [26, 27]. Rs34097845 (-129 G/A) is another SNP located at the promoter region of MPO gene, which has been proved to be associated with myocardial infarction risk [28]. However, the effects of these two polymorphisms on risk of $\mathrm{AD}$ remained unclear.

A case-control study was performed to investigate the association of MPO $\operatorname{rs} 2333227 \quad(-463 \mathrm{~A} / \mathrm{G})$ and rs34097845 (-129 G/A) with AD risk in Chinese Han population.

\section{RESULTS}

\section{The basic characteristics of subjects in this study}

The demographic information of subjects in the case and control groups was showed in Table 1. The case group included 46 males and 70 females with the mean age of $67.39 \pm 9.18$ years old, while there were 56 males and 78 females with the mean age of $68.17 \pm 8.97$ in healthy control group. The case and control groups were matched in gender and age ( $P>0.05$ for both). In this study, the age of onset in AD patients was $63.48 \pm 4.51$ years old. Neither body mass index (BMI) nor tobacco consumption was the influence factor of $\mathrm{AD}$ risk in this population ( $P=0.163$ and 0.169 respectively). However, the percentage of drinker in AD group was obviously higher than that in controls $(37.07 \%$ vs. $24.63 \%$, $P=0.033$ ), indicating drinking might be a risk factor of
AD. In $\mathrm{AD}$ group, $8.62 \%$ cases were diabetes patients, and the ratio in control group was $4.48 \%$, but there was no significant difference $(P=0.182)$. The distribution of hypertension was significantly different between AD and healthy groups $(P=0.037)$.

\section{HWE test}

Distributions of rs2333227 and rs34097845 SNPs genotypes and alleles were confirmed to HWE test both in cases and controls (Table 2, $P>0.05$ for all), indicating the good representativeness of our study population.

\section{Association between $M P O$ polymorphisms and AD risk}

The sequenced results showed the data between PCR-RFLP and sequencing analyses were consistent. Thus, the genotyping results for $M P O$ polymorphisms obtained via PCR-RFLP were reliable.

The frequencies of $\mathrm{GA}$ and $\mathrm{AA}$ genotypes in rs2333227 polymorphism were significantly higher in AD patients than that in healthy controls ( $P>0.05$ for both). In comparison with GG genotype, AA and GA genotypes had no significant association with the risk of AD (Table 2, $P>0.05$ for both). Besides, AA+GA genotypes was associated with increased risk of $\mathrm{AD}(P=0.042, \mathrm{OR}=1.719$, $95 \% \mathrm{CI}=1.017-2.906)$. Meanwhile, A allele was frequently observed in case group, suggesting that A allele might be significantly related to $\mathrm{AD}$ risk $(P=0.041, \mathrm{OR}=1.582$, $95 \% \mathrm{CI}=1.016-2.463)$. For the rs34097845, GA frequencies between $\mathrm{AD}$ patients and the controls were significantly different $(P=0.048)$. GA genotype was correlated to decreased risk of $\mathrm{AD}(\mathrm{OR}=0.555,95 \% \mathrm{CI}=0.308-0.998)$. Additionally, rs34097845 also showed obvious association with decreased risk of $\mathrm{AD}$ under $\mathrm{AA}+\mathrm{GA}$ vs. GG model $(P=0.042, \quad \mathrm{OR}=0.552, \quad 95 \% \mathrm{CI}=0.310-0.983)$. Lower frequency of A allele was discovered in $\mathrm{AD}$ patients, however, it had no significant association with AD risk $(P>0.05)$.

\section{The analysis of haplotype consisted of $M P O$ rs2333227, rs34097845 in AD risk}

The strong linkage disequilibrium was found between rs2333227 and rs34097845 polymorphisms, and haplotypes G-G, A-G and G-A were detected in this study population, but not G-A haplotpye. The frequencies of haplotypes in $\mathrm{AD}$ patients and the controls were showed in Table 3 and the data were $65.52 \%, 23.71 \%, 10.77 \%$ in the case group and $66.79 \%, 16.42 \%, 16.79 \%$ in the control group respectively. But there was no significant difference between the two groups in haplotype frequency, so they did not show obvious association with AD risk. 
Table 1: The demographic characteristics of subjects in the case and control groups

\begin{tabular}{|c|c|c|c|}
\hline Characteristics & Case, $n=116$ & Control, $n=134$ & $\boldsymbol{P}$ \\
\hline \multicolumn{4}{|l|}{ Gender } \\
\hline Male/female & $46 / 70$ & $56 / 78$ & 0.732 \\
\hline \multicolumn{4}{|l|}{ Age (year) } \\
\hline Mean age & $67.39 \pm 9.18$ & $68.17 \pm 8.97$ & 0.654 \\
\hline Age of onset & $63.48 \pm 4.51$ & - & \\
\hline \multicolumn{4}{|c|}{ Body mass index $\left(\mathrm{kg} / \mathrm{m}^{2}\right)$} \\
\hline Mean value & $25.32 \pm 6.35$ & $24.57 \pm 5.51$ & 0.163 \\
\hline \multicolumn{4}{|c|}{ Tobacco consumption $(\%)$} \\
\hline Yes & $38(32.76)$ & $32(23.88)$ & 0.119 \\
\hline \multicolumn{4}{|l|}{ Drinking (\%) } \\
\hline Yes & $43(37.07)$ & $33(24.63)$ & 0.033 \\
\hline \multicolumn{4}{|l|}{ Diabetes $(\%)$} \\
\hline Yes & $10(8.62)$ & $6(4.48)$ & 0.182 \\
\hline \multicolumn{4}{|l|}{ Hypertension (\%) } \\
\hline Yes & $23(19.83)$ & $14(10.45)$ & 0.037 \\
\hline
\end{tabular}

\section{MPO polymorphisms influenced the plasma MPO concentration}

Plasma concentration of MPO were $326.50 \pm 52.28$ and $276.01 \pm 24.87 \mu \mathrm{g} / \mathrm{L}$ respectively in $\mathrm{AD}$ patients and healthy controls. MPO concentration in different genotypes were listed in Table 4. In case group, AA genotype of rs2333227 had lower MPO concentration than GG and GA genotypes $(P<0.01)$, GA genotype also exhibited low MPO concentration compared with

Table 2: Association between $M P O$ polymorphisms and AD risk

\begin{tabular}{lccccc}
\hline Genotype/allele & Case $\mathbf{n}=\mathbf{1 1 6}(\mathbf{\%})$ & Control $\mathbf{n}=\mathbf{1 3 4}(\mathbf{\%})$ & Models & $\boldsymbol{P}$ & OR(95\%CI) \\
\hline rs2333227 & & & & & \\
GG & $68(58.62)$ & $95(70.90)$ & AA vs. GG & 0.262 & $1.956(0.596-6.423)$ \\
GA & $41(35.34)$ & $34(25.37)$ & GA vs. GG & 0.063 & $1.685(0.971-2.923)$ \\
AA & $7(6.03)$ & $5(3.73)$ & AA+GA vs. GG & 0.042 & $1.719(1.017-2.906)$ \\
G & $177(76.29)$ & $224(83.58)$ & AA vs. GA+GG & 0.396 & $1.657(0.511-5.369)$ \\
A & $55(23.71)$ & $44(16.42)$ & A vs. G & 0.041 & $1.582(1.016-2.463)$ \\
$P_{\text {HWE }}$ & 0.805 & 0.382 & & & \\
rs34097845 & & & & & \\
GG & $92(79.31)$ & $91(67.91)$ & AA vs. GG & 0.623 & $0.495(0.044-5.550)$ \\
GA & $23(19.83)$ & $41(30.60)$ & GA vs. GG & 0.048 & $0.555(0.308-0.998)$ \\
AA & $1(0.86)$ & $2(1.49)$ & AA+GA vs. GG & 0.042 & $0.552(0.310-0.983)$ \\
G & $207(89.22)$ & $223(83.21)$ & AA vs. GA+GG & 1.0 & $0.574(0.051-6.412)$ \\
A & $25(10.78)$ & $45(16.79)$ & A vs. G & 0.053 & $0.598(0.354-1.011)$ \\
$P_{\text {HWE }}$ & 0.738 & 0.272 & & & \\
\hline
\end{tabular}

Note: HWE: Hardy-Weinberg equilibrium. 
Table 3: The haplotype analysis of $M P O$ polymorphisms in AD risk

\begin{tabular}{|c|c|c|c|c|}
\hline \multirow[t]{2}{*}{ rs2333227-rs34097845 } & \multicolumn{2}{|c|}{ Haplotype } & \multirow[t]{2}{*}{$P$} & \multirow[t]{2}{*}{ OR $(95 \% C I)$} \\
\hline & Case, $2 n=232(\%)$ & Control, $2 \mathrm{n}=268(\%)$ & & \\
\hline $\mathrm{G}-\mathrm{G}$ & $152(65.52)$ & $179(66.79)$ & - & Ref. \\
\hline A-G & $55(23.71)$ & $44(16.42)$ & 0.092 & $1.472(0.937-2.312)$ \\
\hline G-A & $25(10.77)$ & 45 (16.79) & 0.118 & $0.654(0.383-1.117)$ \\
\hline
\end{tabular}

Table 4: Influences of MPO polymorphisms for plasma MPO concentration

\begin{tabular}{lcc}
\hline Genotypes & Control $($ mean \pm SD, $\boldsymbol{\mu g} / \mathbf{L})$ & Case $(\mathbf{m e a n} \pm \mathbf{S D}, \boldsymbol{\mu g} / \mathbf{L})$ \\
\hline Total & $276.01 \pm 24.87$ & $326.50 \pm 52.28^{\&}$ \\
rs 2333227 & & \\
GG & $283.03 \pm 17.36$ & $350.88 \pm 42.71^{\&}$ \\
GA & $262.71 \pm 27.62^{*}$ & $299.22 \pm 40.83^{* \&}$ \\
AA & $233.2 \pm 47.19^{*}$ & $249.43 \pm 46.49^{* \#}$ \\
rs34097845 & & \\
GG & $271.16 \pm 20.06$ & $317.60 \pm 51.80^{\&}$ \\
GA & $286.98 \pm 31.13^{*}$ & $361.65 \pm 39.56^{* \&}$ \\
AA & $272 \pm 12.73$ & 337 \\
\hline
\end{tabular}

Notes: *, compared with major allele homozygote $P<0.01$; \#, compared with heterozygote $P<0.01$; \&, compared with controls $P<0.01$. According to a Bonferroni correction, $P<0.05 / 2=0.025$ was the significant level.

the GG genotype $(P<0.01)$. In the control group, GA and AA genotype carriers respectively had lower MPO concentration $(P<0.01)$. Significant differences existed in the MPO concentration between the case and control groups respectively in GA and GG genotypes $(P<0.01)$, but AA genotype carriers in case and control groups exhibited similar level of MPO. Rs34097845 GA genotype carriers both in AD and healthy control groups had higher MPO concentrations than those GG genotype carriers $(P<0.01)$. Significant differences of MPO concentration were observed in GG and GA genotype carriers between the cases and controls. AA genotype of rs34097845 SNP had no distinct influence on plasma MPO concentration.

\section{DISCUSSION}

$\mathrm{AD}$ is a chronic neurodegenerative disease, without effective treatment. Thus, prevention is pivotal for the patients. Until now, the etiology of AD still can not be well understood. The initiation of $\mathrm{AD}$ is a consequence of synergistic effect of multiple factors, including genetic and environmental factors. Up to now, a number of risk factors are confirmed for AD, such as obesity, depression, tobacco consumption, drinking abuse, low degree of education, lack of exercise, hypertension, etc. However, in clinic, only a small part of people expose to these factors will finally develop AD, suggesting individual susceptibility to AD. Growing evidences have demonstrated that genetic alterations may be responsible for the individual susceptibility to AD [29]. In addition to $\beta$-amyloid (A $\beta$ ) and Tau protein, there are a large number of genetic molecules which may take part in progression of AD. To investigate the AD-related SNPs may provide a new insight into the etiology of the disease [30].

In present study, we investigated the associated of MPO polymorphisms with risk of $\mathrm{AD}$ in Chinese Han population. The results indicated that rs2333227 SNP was obviously associated with AD risk under five genetic models. In AA+GA vs. GG model, rs2333227 SNP obviously promoted 1.719 times risk of $\mathrm{AD}$. Besides, 1.582 times risk of $\mathrm{AD}$ was presented by the carriage of rs2333227 A allele, compared with $\mathrm{G}$ allele. Under other genetic models, rs2333227 SNP slightly elevated the risk of AD. This result was accorded with previous studies [31-33]. But Combarros and Usui had reported that rs2333227 SNP was not related to $\mathrm{AD}$ risk [34, 35]. Negative association was discovered between rs34097845 SNP and AD risk. Compared with GG genotype, GA genotype and AA+GA genotypes carriers respectively were 0.555 and 0.552 times risk of $\mathrm{AD}$. This was the first study to focus on the association between rs34097845 SNP and AD risk in Chinese Han population. But in the association study of coronary artery disease (CAD), rs34097845 exhibited no association with CAD severity and it did not affect serum MPO activity [36]. 
MPO concentration in plasma was significantly different between $\mathrm{AD}$ patients and healthy controls [24]. In current study, obviously high MPO concentration was observed in $\mathrm{AD}$ patients, this result was consistent with previous study [24]. It has been reported that rs $2333227 \mathrm{~A}$ allele significantly reduced the transcriptional level of MPO [37], thus we speculated that this SNP might be related to the plasma concentration of MPO. Then we found that MPO concentration was lowest in rs2333227 AA genotype carriers, and GG genotype carriers had the highest concentration. Moreover, AD patients with GG and GA genotypes respectively had higher MPO concentration than that in controls, but not AA genotype. A previous study demonstrated that rs2333227 G allele enhanced MPO expression in astrocytes [38]. Rudolph and colleagues found that rs 2333227 AA genotype was correlated to the decreased MPO expression in patients with impaired left ventricular function [37]. We also analyzed the correlation between rs34097845 genotypes and plasma MPO concentration. This results demonstrated that A allele of rs34097845 slightly decreased the MPO concentration in plasma. Based on the data, we hypothesized that rs2333227 and rs34097845 might be involved in etiology of $\mathrm{AD}$ through regulating the transcriptional activity of the gene. It has been reported that abnormal expression of MPO in astrocytes could result in a specific pattern of phospholipid peroxidation and neuronal dysfunction, thus contributing to $\mathrm{AD}$ [38]. However, the molecular mechanisms underlying the functional roles of MPO polymorphisms in AD still remained poorly known. Further investigations will be required.

In addition, the haplotype analysis was also performed in our study. Unfortunately, we did not observe obvious association between MPO haplotype and AD risk based on our study subjects. Several reasons might be responsible for the results. First, the relatively small sample size might be responsible for the abnormal results. Second, the inappropriate reference might be a key cause of the unusual results. G allele of rs2333227 SNP was a relatively protective factor for $\mathrm{AD}$, while $\mathrm{G}$ allele of rs34097845 polymorphism could increase the risk of AD. Thus, G-G might be not an appropriate reference in haplotype analysis of $M P O$ polymorphisms in AD. The analysis of haplotype consisted of MPO rs2333227, rs34097845 in AD risk needed further investigations.

$M P O$ polymorphisms in the promoter region are significantly associated with AD risk via regulating MPO expression in Chinese Han population. Nevertheless, several limitations exists in current study. The sample size is not large enough and the study group only includes one Chinese Han population. Moreover, the interaction of $M P O$ polymorphisms with other factors such as environmental factors was not considered. In view of these reasons, well designed studies with large sample size and multiple populations are necessary to certify the role of $M P O$ promoter polymorphisms in $\mathrm{AD}$ in the future.

\section{MATERIALS AND METHODS}

\section{Subjects}

Between 2014 January and 2016 January, 116 AD patients and 134 healthy controls were recruited from Tianjin Huanhu Hospital as the case and control groups respectively. All subjects were Chinese Han population without any blood connection. AD patients were diagnosed by two pathologists according to the diagnosis criteria [39]. Healthy controls were matched with the cases in age and gender. Any person who had other neurodegenerative diseases, vascular diseases or systemic diseases would be omitted from this study.

This case-control study was approved by the Ethnic Committee of Tianjin Huanhu Hospital. All of the participants signed the written informed consents.

\section{Sample collection}

A total of $2 \mathrm{ml}$ fasting venous blood was collected from each subject in the morning using blood collection tube with EDTA. Blood samples were centrifuged into leukocytes and plasma, and then stored at $-80^{\circ} \mathrm{C}$.

\section{Genotyping for $M P O$ polymorphisms}

Genomic DNA was extracted from leukocytes using the traditional phenol chloroform isoamyl alcohol method. MPO rs2333227 and rs34097845 polymorphisms were genotyped using polymerase chain reactionrestriction fragment length polymorphism (PCR-RFLP) method following previous studies [40]. Each test was repeated three times. Both of these two polymorphisms were determined GG, AG or AA genotype in selected subjects. $20 \%$ samples were randomly selected to confirm the genotyping results via the ABI 3700 DNA analyzer (Applied BioSystem, USA).

\section{Detection of MPO concentration}

Plasma MPO concentration was detected by enzyme-linked immuno sorbent assay (ELISA) method using a human myeloperoxidase, MPO ELISA Kit (Jianglai Biotechnology, Shanghai) according to the manufacturer's instruction. Each test was performed in triplicate, and the average value was used for the final analysis.

\section{Statistical analysis}

Hardy-Weinberg equilibrium (HWE) test was used to detect the representativeness of the subjects. Genotype distributions between cases and controls were compared by Chi square test or Fisher's exact test. Odds 
ratios (ORs) with 95\% confidence intervals (CIs) were utilized to estimate the relative risk of AD. The linkage disequilibrium of $M P O$ two polymorphisms was analyzed by Haploview software. Plasma MPO concentration was assessed by non-parametric test (Mann-Whitney U test), meanwhile, the significant level was adjusted by Bonferroni method. All the calculations were performed by PASW 18.0. $P<0.05$ was considered as significant.

\section{Author contributions}

Wenzhen Ji, Yu Zhang conceived and designed the experiments; Yu Zhang conceived and performed the experiments; Yu Zhang prepared figures. Yu Zhang wrote the main manuscript text. All authors reviewed the manuscript.

\section{CONFLICTS OF INTEREST}

The authors declare that they have no competing interest.

\section{REFERENCES}

1. Szeto JY, Lewis SJ. Current Treatment Options for Alzheimer's Disease and Parkinson's Disease Dementia. Curr Neuropharmacol. 2016; 14: 326-38.

2. Niu H, Alvarez-Alvarez I, Guillen-Grima F, AguinagaOntoso I. Prevalence and incidence of Alzheimer's disease in Europe: A meta-analysis. Neurologia. 2017; 32: 523-32. https://doi.org/10.1016/j.nrl.2016.02.016.

3. Perez Romero A, Gonzalez Garrido S. The importance of behavioural and pyschological symptoms in Alzheimer's disease. Neurologia. 2016 Apr 26. https://doi.org/10.1016/j. nrl.2016.02.024. [Epub ahead of print].

4. Keogh-Brown MR, Jensen HT, Arrighi HM, Smith RD. The Impact of Alzheimer's Disease on the Chinese Economy. EBioMedicine. 2016; 4: 184-90. https://doi.org/10.1016/j. ebiom.2015.12.019.

5. Kazhungil F, Velayudhan R, Kumar M, Thazhe Mangool R. A comparative study of caregiver burden in late-onset depression and Alzheimer's disease. Psychogeriatrics. 2016; 16: 209-15. https://doi.org/10.1111/psyg.12141.

6. Rodriguez-Vieitez E, Saint-Aubert L, Carter SF, Almkvist O, Farid K, Scholl M, Chiotis K, Thordardottir S, Graff C, Wall A, Langstrom B, Nordberg A. Diverging longitudinal changes in astrocytosis and amyloid PET in autosomal dominant Alzheimer's disease. Brain. 2016; 139: 922-36. https://doi.org/10.1093/brain/awv404.

7. Vella LJ, Hill AF, Cheng L. Focus on Extracellular Vesicles: Exosomes and Their Role in Protein Trafficking and Biomarker Potential in Alzheimer's and Parkinson's Disease. Int J Mol Sci. 2016; 17: 173. https://doi.org/10.3390/ijms17020173.

8. Baloyannis SJ. Brain capillaries in Alzheimer's disease. Hell J Nucl Med. 2015; 18: 152.
9. Hung AS, Liang Y, Chow TC, Tang HC, Wu SL, Wai MS, Yew DT. Mutated tau, amyloid and neuroinflammation in Alzheimer disease-A brief review. Prog Histochem Cytochem. 2016; 51: 1-8. https://doi.org/10.1016/j.proghi.2016.01.001.

10. Sweeney MD, Ayyadurai S, Zlokovic BV. Pericytes of the neurovascular unit: key functions and signaling pathways. Nat Neurosci. 2016; 19: 771-83. https://doi.org/10.1038/nn.4288.

11. Mahley RW. Apolipoprotein E: from cardiovascular disease to neurodegenerative disorders. J Mol Med (Berl). 2016; 94 : 739-46. https://doi.org/10.1007/s00109-016-1427-y.

12. Piras A, Collin L, Gruninger F, Graff C, Ronnback A. Autophagic and lysosomal defects in human tauopathies: analysis of post-mortem brain from patients with familial Alzheimer disease, corticobasal degeneration and progressive supranuclear palsy. Acta Neuropathol Commun. 2016; 4: 22. https://doi.org/10.1186/s40478-016-0292-9.

13. de Wilde MC, Overk CR, Sijben JW, Masliah E. Metaanalysis of synaptic pathology in Alzheimer's disease reveals selective molecular vesicular machinery vulnerability. Alzheimers Dement. 2016; 12: 633-44. https://doi.org/10.1016/j.jalz.2015.12.005.

14. Hohman TJ, Bush WS, Jiang L, Brown-Gentry KD, Torstenson ES, Dudek SM, Mukherjee S, Naj A, Kunkle BW, Ritchie MD, Martin ER, Schellenberg GD, Mayeux R, et al. Discovery of gene-gene interactions across multiple independent data sets of late onset Alzheimer disease from the Alzheimer Disease Genetics Consortium. Neurobiol Aging. 2016; 38: 141-50. https://doi.org/10.1016/j. neurobiolaging.2015.10.031.

15. Yoon SY, Kim DH. Alzheimer's disease genes and autophagy. Brain Res. 2016; 1649: 201-9. https://doi. org/10.1016/j.brainres.2016.03.018.

16. Hulsdunker J, Zeiser R. In Vivo Myeloperoxidase Imaging and Flow Cytometry Analysis of Intestinal Myeloid Cells. Methods Mol Biol. 2016; 1422: 161-7. https://doi. org/10.1007/978-1-4939-3603-8_15.

17. Perianayagam MC, Tighiouart $\mathrm{H}$, Liangos $\mathrm{O}$, Kouznetsov D, Wald R, Rao F, O'Connor DT, Jaber BL. Polymorphisms in the myeloperoxidase gene locus are associated with acute kidney injury-related outcomes. Kidney Int. 2012; 82: 90919. https://doi.org/10.1038/ki.2012.235.

18. Rensen SS, Bieghs V, Xanthoulea S, Arfianti E, Bakker JA, Shiri-Sverdlov R, Hofker MH, Greve JW, Buurman WA. Neutrophil-derived myeloperoxidase aggravates nonalcoholic steatohepatitis in low-density lipoprotein receptordeficient mice. PLoS One. 2012; 7: e52411. https://doi. org/10.1371/journal.pone.0052411.

19. Soubhye J, Aldib I, Delporte C, Prevost M, Dufrasne F, Antwerpen PV. Myeloperoxidase as a Target for the Treatment of Inflammatory Syndromes: Mechanisms and Structure Activity Relationships of Inhibitors. Curr Med Chem. 2016; 23: 3975-4008.

20. Lehners A, Lange S, Niemann G, Rosendahl A, MeyerSchwesinger C, Oh J, Stahl R, Ehmke H, Benndorf 
R, Klinke A, Baldus S, Wenzel UO. Myeloperoxidase deficiency ameliorates progression of chronic kidney disease in mice. Am J Physiol Renal Physiol. 2014; 307: F407-17. https://doi.org/10.1152/ajprenal.00262.2014.

21. Laura RP, Dong D, Reynolds WF, Maki RA. T47D Cells Expressing Myeloperoxidase Are Able to Process, Traffic and Store the Mature Protein in Lysosomes: Studies in T47D Cells Reveal a Role for Cys319 in MPO Biosynthesis that Precedes Its Known Role in Inter-Molecular Disulfide Bond Formation. PLoS One. 2016; 11: e0149391. https:// doi.org/10.1371/journal.pone.0149391.

22. Lu N, Ding $\mathrm{Y}$, Tian $\mathrm{R}$, Peng YY. Inhibition of myeloperoxidase-mediated oxidative damage by nitrite in SH-SY5Y cells: Relevance to neuroprotection in neurodegenerative diseases. Eur J Pharmacol. 2016; 780: 142-7. https://doi.org/10.1016/j.ejphar.2016.03.042.

23. Lefkowitz DL, Lefkowitz SS. Microglia and myeloperoxidase: a deadly partnership in neurodegenerative disease. Free Radic Biol Med. 2008; 45: 726-31. https://doi. org/10.1016/j.freeradbiomed.2008.05.021.

24. Tzikas S, Schlak D, Sopova K, Gatsiou A, Stakos D, Stamatelopoulos K, Stellos K, Laske C. Increased myeloperoxidase plasma levels in patients with Alzheimer's disease. J Alzheimers Dis. 2014; 39: 557-64. https://doi. org/10.3233/JAD-131469.

25. Rudolph V, Rudolph TK, Kubala L, Clauberg N, Maas R, Pekarova M, Klinke A, Lau D, Szocs K, Meinertz T, Boger RH, Baldus S. A myeloperoxidase promoter polymorphism is independently associated with mortality in patients with impaired left ventricular function. Free Radic Biol Med. 2009; 47: 1584-90. https://doi.org/10.1016/j. freeradbiomed.2009.09.001.

26. Piedrafita FJ, Molander RB, Vansant G, Orlova EA, Pfahl M, Reynolds WF. An Alu element in the myeloperoxidase promoter contains a composite SP1-thyroid hormone-retinoic acid response element. J Biol Chem. 1996; 271: 14412-20.

27. Hansson M, Olsson I, Nauseef WM. Biosynthesis, processing, and sorting of human myeloperoxidase. Arch Biochem Biophys. 2006; 445: 214-24. https://doi. org/10.1016/j.abb.2005.08.009.

28. Zotova E, Lyrenas L, de Faire U, Morgenstern R, Gigante B, Bennet AM. The myeloperoxidase gene and its influence on myocardial infarction in a Swedish population: protective role of the $-129 \mathrm{~A}$ allele in women. Coron Artery Dis. 2009; 20: 322-6. https://doi.org/10.1097/ MCA.0b013e32832da06d.

29. Berardelli A, Wenning GK, Antonini A, Berg D, Bloem BR, Bonifati V, Brooks D, Burn DJ, Colosimo C, Fanciulli A, Ferreira J, Gasser T, Grandas F, et al. EFNS/MDS-ES/ ENS [corrected] recommendations for the diagnosis of Parkinson's disease. Eur J Neurol. 2013; 20: 16-34. https:// doi.org/10.1111/ene.12022.

30. Videm V, Olsen GD. No relationship between neutrophil granulocyte activation and the myeloperoxidase gene - 129 $\mathrm{G}>\mathrm{A}$ and $-463 \mathrm{G}>\mathrm{A}$ promoter polymorphisms: implications for investigations of cardiovascular disease. Coron Artery Dis. 2009; 20: 446-52.

31. Han Z, Huang H, Gao Y, Huang Q. Functional annotation of Alzheimer's disease associated loci revealed by GWASs. PLoS One. 2017; 12: e0179677. https://doi.org/10.1371/ journal.pone.0179677.

32. Huang P, Hsieh SW, Chang YH, Hour AL, Chen HY, Liu CK. Differences in the frequency of Alzheimer's diseaseassociated genomic variations in populations of different races. Geriatr Gerontol Int. 2017 Jul 4. https://doi. org/10.1111/ggi.13059. [Epub ahead of print].

33. Leininger-Muller B, Hoy A, Herbeth B, Pfister M, Serot JM, Stavljenic-Rukavina M, Massana L, Passmore P, Siest G, Visvikis S. Myeloperoxidase G-463A polymorphism and Alzheimer's disease in the ApoEurope study. Neurosci Lett. 2003; 349: 95-8.

34. Zappia M, Manna I, Serra P, Cittadella R, Andreoli V, La Russa A, Annesi F, Spadafora P, Romeo N, Nicoletti G, Messina D, Gambardella A, Quattrone A. Increased risk for Alzheimer disease with the interaction of MPO and A2M polymorphisms. Arch Neurol. 2004; 61: 341-4. https://doi. org/10.1001/archneur.61.3.341.

35. Crawford FC, Freeman MJ, Schinka JA, Morris MD, Abdullah LI, Richards D, Sevush S, Duara R, Mullan MJ. Association between Alzheimer's disease and a functional polymorphism in the Myeloperoxidase gene. Exp Neurol. 2001; 167: 456-9. https://doi.org/10.1006/exnr.2000.7560.

36. Combarros O, Infante J, Llorca J, Pena N, FernandezViadero C, Berciano J. The myeloperoxidase gene in Alzheimer's disease: a case-control study and meta-analysis. Neurosci Lett. 2002; 326: 33-6.

37. Usui C, Shibata N, Ohnuma T, Higashi S, Ohkubo T, Ueki A, Nagao M, Arai H. No genetic association between the myeloperoxidase gene -463 polymorphism and estrogen receptor-alpha gene polymorphisms and Japanese sporadic Alzheimer's disease. Dement Geriatr Cogn Disord. 2006; 21: 296-9. https://doi.org/10.1159/000091437.

38. $\mathrm{Hu} \mathrm{JL}, \mathrm{Xu} \mathrm{JB}$, Zhou X, Jiang TM, Li YM, Zhang M. [Correlation between MPO $129 \mathrm{~A} / \mathrm{G}$ polymorphism and severity of coronary artery disease]. [Article in Chinese]. Zhongguo Ying Yong Sheng Li Xue Za Zhi. 2011; 27: 306-10.

39. Rudolph V, Rudolph TK, Hennings JC, Blankenberg S, Schnabel R, Steven D, Haddad M, Knittel K, Wende $\mathrm{S}$, Wenzel J, Munzel T, Heitzer T, Meinertz T, et al. Activation of polymorphonuclear neutrophils in patients with impaired left ventricular function. Free Radic Biol Med. 2007; 43: 1189-96. https://doi.org/10.1016/j. freeradbiomed.2007.07.016.

40. Maki RA, Tyurin VA, Lyon RC, Hamilton RL, DeKosky ST, Kagan VE, Reynolds WF. Aberrant expression of myeloperoxidase in astrocytes promotes phospholipid oxidation and memory deficits in a mouse model of Alzheimer disease. J Biol Chem. 2009; 284: 3158-69. https://doi.org/10.1074/jbc.M807731200. 